\title{
Hydrate formation in natural gas pipelines
}

\author{
M. Naseer \& W. Brandstätter \\ Department of Petroleum Production and Processing, \\ University of Leoben, Austria
}

\begin{abstract}
Gas hydrates pose a problem to the flow assurance programs in the oil and gas industry. This paper highlights the mechanisms of hydrate formation in natural gas pipelines. Computational Fluid Dynamics (CFD) was used to gain insight in to the mechanisms of water vapour condensation and consequent water accumulation in the lowered sections of a gas pipeline. The pipeline temperature profile, condensation of water vapour at walls, hydrate formation and hydrate slurry rheology were modelled with user defined functions (UDF) integrated into the CFD-software Fluent. It was found that the uphill sections of gas pipelines are natural sites for water accumulation and hydrate formation. It is demonstrated that CFD is a valuable tool to understand complex physical phenomena occurring in multiphase flow problems.
\end{abstract}

Keywords: natural gas, pipelines, condensation, hydrate formation, uphill sections, viscosity, nucleation, sagging sections, kinetics, macroscopic.

\section{Introduction}

The growth of energy demand is constantly pushing the gas producers to operate at higher pressures. In the start of the twentieth century, the natural gas industry started facing the problem of unexpected pipeline blockage. It was shown in 1934 by HammerSchmidt that the blockages appearing to be ice were actually gas hydrates [1]. The gas hydrate formation in oil and gas pipelines is an undesirable situation, undermining the flow assurance programs in addition to posing threat to the personnel and equipment. Clathrate hydrates are non-stoichiometric mixtures of water and natural gas in which the gas molecules are trapped in polygonal crystalline structure made of water molecules [2]. The water molecules arrange themselves in an orderly fashion around the gas molecules, thus entrapping them. 
These gas hydrates have the appearance of ice but can form well above the freezing point of water at high pressures.

Hydrate formation in natural gas pipelines is a complex phenomenon to capture in its entirety. The sequence of events leading to hydrate formation in gas pipelines involves water vapor condensation, accumulation of water at lowered sections of the pipeline, nucleation and growth of hydrate particles ultimately blocking the pipeline. The site of pipeline blockage may not be the same as that of hydrate deposition because just at the beginning of summer, the hydrate just released from the pipewall along the length may migrate downstream and deposit at sagging sections of the pipeline to cause the blockage [3]. Experiments on natural gas hydrate under actual operating conditions in pipelines is difficult to emulate due to scale of the problem and high pressures involved. However, different experimenters have focused on various aspects of the hydrate formation. Bishoni et al. [4] presented the formation and decomposition kinetics of gas hydrates at a conceptual level, highlighting the different phases of hydrate formation and growth. Riberio et al. [5] provided a critical review of literature regarding hydrate formation kinetics, highlighting the modeling efforts by different researchers. In an effort to emulate actual hydrate formation conditions, industrial scale flow loop experiments were setup by ExxonMobil Upstream Research Company [6] and Tulsa University [7] and Davies et al. [8] validated their hydrate formation model against the experimental data. While the hydrate formation model just mentioned was for the oil dominated flowlines, the hydrate formation may be quite different in natural gas flowlines.

\section{Theory}

Natural gas pipelines always have some moisture content as the gas from the formations reach the well head saturated with water [3]. During the cold season, the ground temperatures are lower resulting in the temperature drop of the pipeline content. If the temperature of the gas falls below the saturation temperature corresponding to the dew point of water, condensation of water vapors will start. In straighter sections of the pipeline, the condensate starts to accumulate at the base of the pipe due to gravity. The accumulation may increase at the base of the sagging/ uphill sections of the pipe. If enough water is present and the thermodynamic conditions are suitable, hydrate nucleation processes are initiated. To analyse hydrate formation on a theoretical basis in principle two choices exist: onedimensional (1-D) or three-dimensional (3-D) models. While 1-D CFD models may be suitable for very long pipeline sections, the effects of water condensation and accumulation can only be modelled using the 3-D CFD methods.

\section{CFD model}

As an application example the pipeline section depicted in figure 1 was selected. The computational domain consists of approximately 84000 polyhedral control 


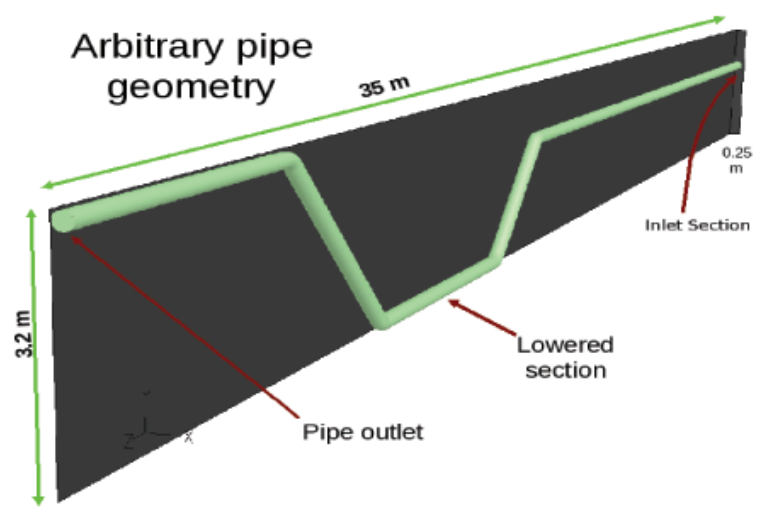

Figure 1: Natural gas pipeline.

volumes. The grid was refined during the computational run time to resolve the accumulation phenomena.

The "Mixture Model" of Fluent [9] was used to represent the interpenetrating continua consisting of mixture components.

Table 1: Model parameters.

\begin{tabular}{|c|r|r|}
\hline No. & Model parameter & Value \\
\hline 1. & Pipe diameter & $0.25 \mathrm{~m}$ \\
2. & Inlet velocity & $2 \mathrm{~m} / \mathrm{s}$ \\
3. & Water vapor volume fraction & 0.2 \\
4. & Inlet temperature & $315 \mathrm{~K}$ \\
5. & Operating pressure & $6.3 \mathrm{Mpa}$ \\
\hline
\end{tabular}

Table 1 depicts different parameters of the pipeline section. The Pressure Implicit Split Operator (PISO) algorithm was used to couple the pressure and velocity fields. The K-Epsilon model was included to model the effects of turbulence.

\subsection{Mathematical framework}

The mixture model treats ' $n$ ' different phases as interpenetrating and models the fluid flow with conservation equations of mass, momentum and energy. The continuity, momentum and energy equations for the mixture in differential form [9] are given below. 
Continuity:

$$
\frac{\partial}{\partial t}\left(\rho_{m}\right)+\nabla \cdot\left(\rho_{m} \vec{v}_{m}\right)=0
$$

Momentum:

$$
\begin{aligned}
\frac{\partial}{\partial t}\left(\rho_{m} \vec{v}_{m}\right)+\nabla \cdot\left(\rho_{m} \vec{v}_{m} \vec{v}_{m}\right)= & \nabla p+\nabla \cdot\left[\mu_{m}\left(\nabla \vec{v}_{m}+\nabla \vec{v}_{m}^{T}\right)+\rho_{m} \vec{g}\right. \\
& +\vec{F}+\nabla \cdot\left(\sum_{k=1}^{n} \alpha_{k} \rho_{k} \vec{v}_{d r, k} \vec{v}_{d r, k}\right)
\end{aligned}
$$

Energy:

$$
\frac{\partial}{\partial t} \sum_{k=1}^{n}\left(\alpha_{k} \rho_{k} E_{k}\right)+\nabla \cdot \sum_{k=1}^{n}\left(\alpha_{k} \vec{v}_{k}\left(\rho_{k} E_{k}+p\right)\right)=\nabla \cdot\left[k_{e f f} \nabla T\right]+S_{E}
$$

where $\vec{v}_{m}$ is the mass averaged velocity, $\rho_{m}$ and $\mu_{m}$ are volume fraction averaged mixture density and viscosity respectively, $\vec{v}_{d r, k}$ is the drift velocity of individual phases, $E_{k}$ is the energy of phase $k, \alpha_{k}$ represents the volume fraction of phase $k$, $p$ represents pressure, $k_{\text {eff }}$ is the effective thermal conductivity, $T$ represents the temperature and $S_{E}$ represents energy source. In order to model the drag of the primary phase on the secondary phases, a drift flux model available in the Fluent was used where, the slip velocity was modelled as provided by Minnan et al. [9] and the drag function correlation of Schiller and Neumann $[9,10]$ was used.

\subsection{Model setup}

For the sake of this study, a certain composition of natural gas was considered at the inlet to the pipe. The molar concentrations were used as input to the program ' $\mathrm{CSM}$ Hyd' [2] and a pressure-temperature equilibrium curve for the hydrate formation was obtained. Based on the best fit through the equilibrium data, an equation was obtained representing the threshold temperature for hydrate formation as

$$
T=8.5274 \times \ln (P)+270.86
$$

Where $T$ is Temperature in Kelvin and $P$ is the pressure in MPa.

To calculate the saturation temperature according to the partial pressure of vapor, the following equation was used.

$$
T_{\text {sat }}=16.335 \times \ln (P)+167.08
$$

In order to accurately model the condensation in Fluent, a relationship developed by De Schepper et al. [11,12] was employed.

$$
M_{\text {water }}=0.1 \times \alpha_{\text {vapour }} \times \rho_{\text {vapour }}\left|T_{\text {sat }}-T_{\text {wall }}\right| / T_{\text {sat }}
$$

Modelling the temperature profile of the pipeline content along the length of the buried pipeline is a challenging task because it varies with the seasonal changes 
and also with the local changes in the conditions in the vicinity of the pipeline. A simplified expression was used [13] to describe the pipe temperature profile along the length.

$$
T(x)=T_{G r}+\left(T_{o}-T_{G r}\right) \exp \left(\frac{-k \pi D x}{0.28 Q C_{p}}\right)
$$

where $T_{G r}$ is the ground temperature, $x$ is the distance along the pipe from the inlet, $D$ is the pipe diameter, $\mathrm{k}$ is thermal conductivity, $Q$ is the volumetric flow rate and $C_{p}$ is the specific heat capacity.

The kinetic modeling of hydrate nucleation and growth requires the definition of a driving force. While different researchers have presented their versions of kinetic models, there is a general lack of consensus among them. The applicability of these models to dynamic flow systems is limited by assumptions regarding thermodynamics at the molecular level and to the specifics of the experimental setup. Most notable among them is the work by Kashchiev and Firoozabadi who considered the difference between chemical potential of the species as the driving force [14].

An authentic kinetic model can serve as bridge between the microscopic phenomenon of crystallization and its macroscopic effects. The assumption is made here that there is an onset of instantaneous nucleation due to high number of nucleation sites (on the pipe wall due to roughness) and high supersaturation of the condensed liquid water film on the pipe wall with hydrocarbon gas at high pressure. Based on the general stoichiometric molar relationship for hydrate formation, the threshold mass fraction (considering water and hydrocarbon gas) in a cell was set to be approximately 0.866 . This threshold value was imposed through the UDFs.

When hydrate formation conditions are reached in a computational cell, the hydrate formation is modelled by mass transfer equations for both the phases i.e. water and gas by the following simplified relationship:

$$
M_{\text {hyd }}=\left(\rho_{\text {water }} \times \alpha_{\text {water }}\right)+\left(\rho_{\text {gas }} \times \alpha_{\text {gas }}\right)
$$

To incorporate the rheological effects of the increase of suspension in the solution, the following relationship was used $[10,15]$ :

$$
\mu_{\text {slurry }}=\alpha_{\text {water }}\left(1-\alpha_{\text {hyd }}\right)^{-2.5}
$$

\section{Results and discussion}

The effect of water condensation at the pipe walls and accumulation at the bottom of the pipe section is depicted in figure 2 . At the onset of condensation of water vapor, the condensed water starts to accumulate at the bottom section of the pipe i.e. at the 6 o'clock position and it flows along the mean flow direction. This effect can be clearly observed in figure 2 . It is asserted that the effect of accumulation can only be highlighted in three dimensional simulations and can be completely overlooked in the one dimensional models. Furthermore, the accumulation of free 

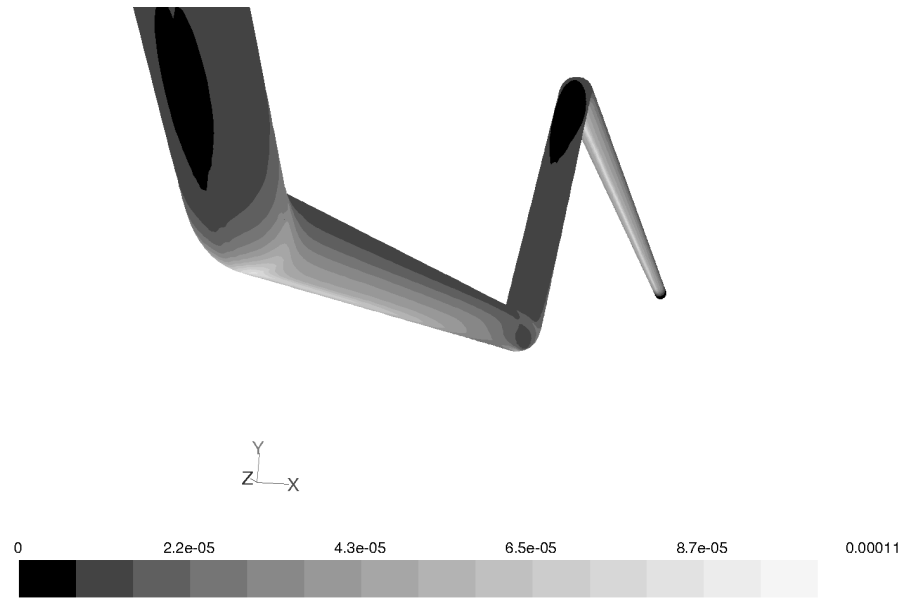

Figure 2: Water phase contours showing condensation at wall and accumulation at the bottom of the pipe.

water at the base of the uphill section of the pipe is displayed. It is concluded that there are two main mechanisms of water accumulation in pipelines due to gravity i.e. accumulation at bottom section of the pipe and accumulation at the base of uphill/ sagging sections of the pipe. The accumulation phenomena is a compound effect taking place at the base of uphill section of the pipe for aforementioned reasons and is clearly visible in figure 2 .

Presence of free water is a key factor in the hydrate formation initiation as argued by Sloan [2]. The simulation shows that the base of the sagging sections of pipelines are most susceptible to hydrate nucleation as these regions serve as stagnation points/ traps for any condensed water in gas flows. The hydrate formation at these sections can start in winters. Multiple hydrate deposits along the length of the pipe result in additional pressure drop. The real problem may start at the beginning of summers, when the hydrate loosens up at the pipeline walls (not completely dissociated) and starts traveling [3]. The sloughed off hydrate then flows along the mean flow direction until it reaches lowered sections and gets trapped as packed deposits.

The region of high hydrate volume fraction in accumulated water is shown in figure 3. The high hydrate volume fraction region is marked by the iso-surfaces of hydrate concentration which can be clearly distinguished from the water isosurfaces. It is evident that the water stagnation points are most susceptible to hydrate formation. The hydrate formation acts as additional resistance to the flow as shown by the velocity vectors in the cut-plane through $x=0$. The velocity vectors actually drawn at $x=0$ iso-plane are offset by some distance for clearer visibility. Stagnation zone with high hydrate volume fraction regions can be observed. In real pipelines the difference in velocities between the mean flow and the hydrate deposits would result in a drag force between phases leading to pressure drop undermining the flow assurance. 


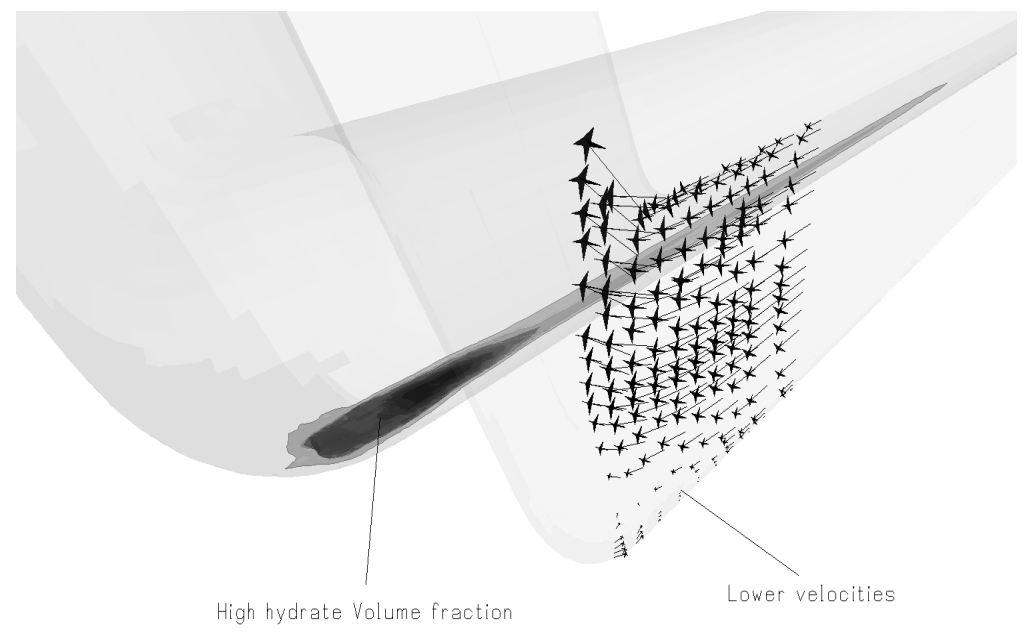

Figure 3: Velocity vectors at $\mathrm{x}=0$ plane drawn at an offset showing the effect of high hydrate concentration.

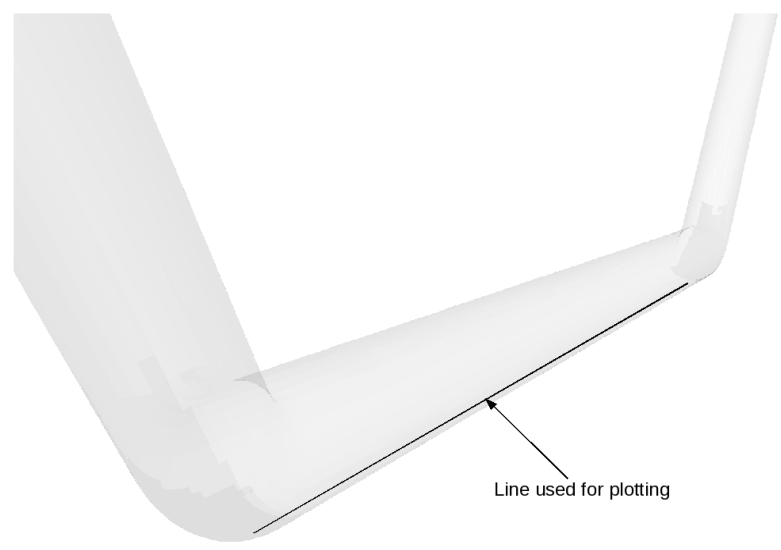

Figure 4: Line used for the plots.

In order to plot different parameters, a line just at the bottom of the lowered section of the pipe in between the sections $22 \mathrm{~m}$ and $28 \mathrm{~m}$ (measured from the inlet) was used as shown in figure 4.

Figure 5 shows the variation of viscosity and water volume fraction at the base of the lowered (straight) section. The gradual increase in viscosity mixture defined as $\mu_{m}=\sum_{k=1}^{n} \alpha_{k} \mu_{k}$ goes hand in hand with increase in the water volume fraction with a sudden spike indicating high hydrate volume fraction influencing the mixture viscosity. The gradual slope in the curve before $27 \mathrm{~m}$ highlights the accumulation phenomena of water (pileup). The sudden increase in the viscosity 


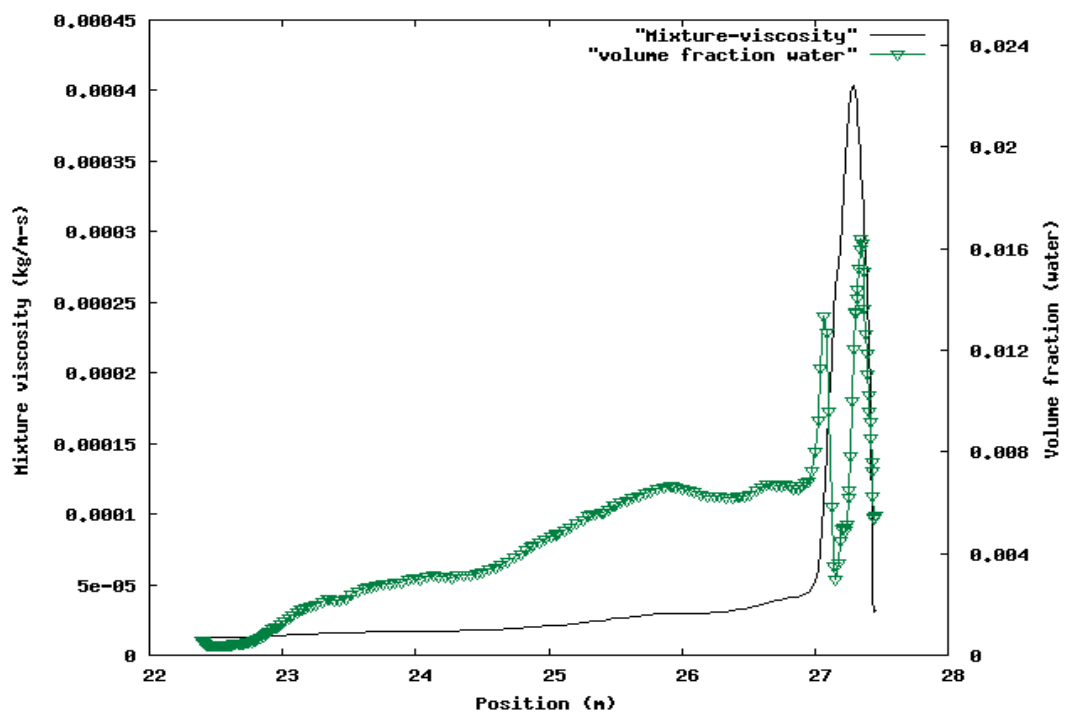

Figure 5: Mixture viscosity and water volume fraction along the length of lowered section of the pipe.

beyond $27 \mathrm{~m}$ is indicative of the formation of a new phase drastically effecting the viscosity of the mixture.

The water volume fraction depicted in figure 5 shows a gradual increase initially followed by a sharp increase, a sharp decrease and again a sharp increase. The sharp decrease belongs to the region of high hydrate volume fraction and is acting as a 'double sided tidal barrage' between down stream and upstream water. It is interesting to note the effect of hydrate deposition on the water film moving in the mean flow direction. At the upstream side of the deposit, it is acting like a wall, resulting in increase in water volume as depicted in the figure and at the downstream side, the result of water accumulation is due to the backflow of water film adjacent to the deposit. The backflow is assisted by loss in the dynamic pressure in the region shadowed by the deposit and gravity effects at the uphill section.

The mixture velocity $\overrightarrow{v_{m}}=\sum_{k=1}^{n} \alpha_{k} \rho_{k} \overrightarrow{v_{k}} / \rho_{m}$ at the bottom of the pipe section is shown in figure 6 . The increase in the velocity between $22-22.5 \mathrm{~m}$ section is due to mean flow from the downhill section impinging the wall resulting in increase in the velocity. Beyond $22.5 \mathrm{~m}$ the mixture velocity drops due to friction at the walls. In the section between $23-26 \mathrm{~m}$, the mixture velocity oscillates with a mean value of about $0.5 \mathrm{~m} / \mathrm{s}$. The "fish-hook" curve beyond $27 \mathrm{~m}$ shows the drastic drop in the mixture velocity due to high hydrate volume fraction.

The variation of static and dynamic pressure is depicted in figure 7 . The drastic increase of static pressure at about $27 \mathrm{~m}$ (and decrease of dynamic pressure) indicates the effects of high hydrate deposition. 


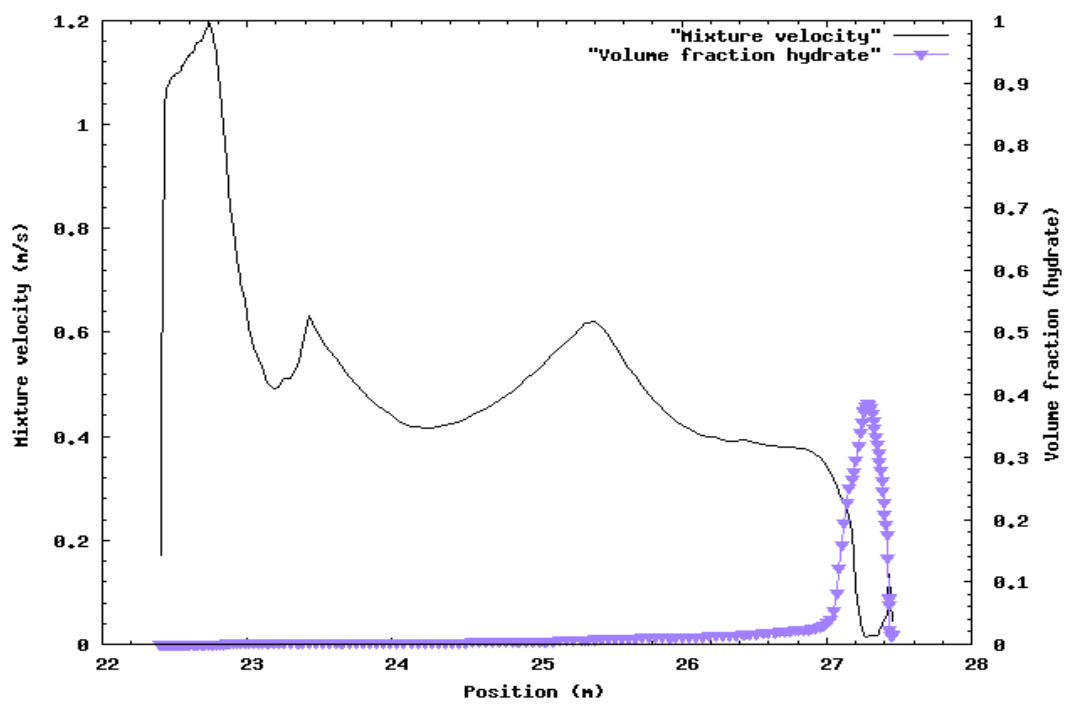

Figure 6: Mixture velocity and hydrate volume fraction along the length of lowered section of the pipe.

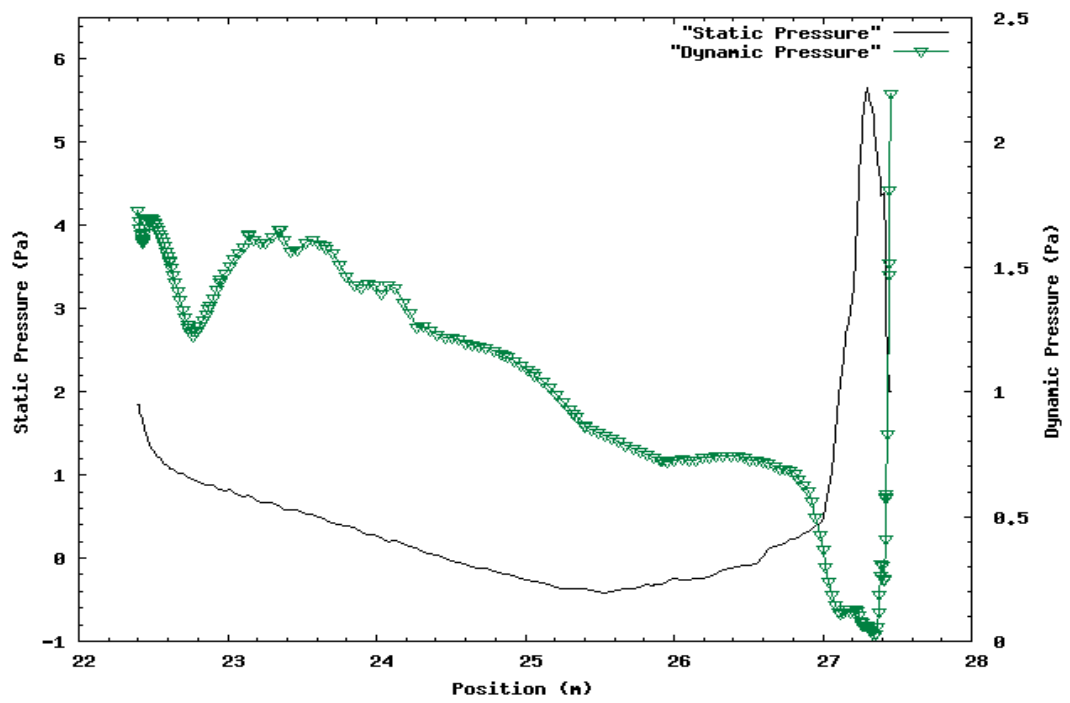

Figure 7: Pressure variation along the length of lowered section of the pipe.

\section{Conclusions and future work}

A 3D-CFD model for hydrate formation in gas pipelines has been developed. The results achieved so far are in agreement with existing knowledge about the condensation and accumulation of liquid water regions due to gravity in 
hilly terrain pipelines. Furthermore critical regions for hydrate formation could be identified. Overall very plausible theoretical explanations about the complex physical phenomenon taking place could be found.

Unfortunately no direct comparison with experimental results due to lack of data so far could be made. However, this will be subject to a forthcoming paper. Measurements for model validation in a real hilly terrain pipeline are currently ongoing. Future work is also aimed at integrating a correct kinetic model to simulate the effect of hydrate nucleation and growth phenomena on a macroscopic scale.

\section{References}

[1] J.J. Carroll, Natural Gas Hydrate: A Guide for Engineers. Elsevier Science, 2003.

[2] E.D. Sloan \& K.A. Koh, Clathrate Hydrates of Natural Gases, 2008.

[3] W.M. Deaton \& E.M. Frost, Jr., Gas hydrates and their relation to the operation of natural-gas pipe lines. Technical report, United States Department of the Interior, Bureau of Mines, July 1946.

[4] P.Raj Bishoni \& V. Natarajan, Formation and decomposition of gas hydrates. Fluid Phase Equilibria, 117, pp. 168-177, 1996.

[5] C.P. Ribeiro Jr. \& P.L.C. Lage, Modelling of hydrate formation kinetics: State-of-the-art and future directions. Chemical Engineering Science, 63(8), pp. 2007-2034, 2008.

[6] UBC, https://circle.ubc.ca/bitstream/handle/2429/1133/5434.pdf.

[7] UT, http://www.tuhfp.utulsa.edu/.

[8] S.R. Davies, J.A. Boxall, L.E. Dieker, A.K. Sum, C.A. Koh, E.D. Sloan, J.L. Creek \& Z.-G. Xu, Predicting hydrate plug formation in oil-dominated flowlines. Journal of Petroleum Science and Engineering, 72(3-4), pp. 302309, 2010.

[9] Fluent 6.3 Users Guide.

[10] B.V. Balakin, A.C. Hoffmann \& P. Kosinki, Experimental study and computational fluid dynamics modeling of deposition of hydrate particles in a pipeline with turbulent water flow. Chemical Engineering Science, 22 November 2010.

[11] S.C.K. De Schepper, G.J. Heynderickx \& G.B. Marin, Modeling the evaporation of a hydrocarbon feedstock in the convection section of a steam cracker. Computers \& Chemical Engineering, 33(1), pp. 122-132, 2009.

[12] A. Alizadehdakhel, M. Rahimi \& A.A. Alsairafi, Cfd modeling of flow and heat transfer in a thermosyphon. International Communications in Heat and Mass Transfer, 37(3), pp. 312-318, 2010.

[13] Y.F. Makogon, Hydrates of Hydrocarbons. PennWell Books, 1997.

[14] D. Kashchiev and A. Firoozabadi, Nucleation of gas hydrates. Journal of Crystal Growth, 243(3-4), pp. 476-489, 2002.

[15] P. Mills, Non-Newtonian behaviour of flocculated suspensions. J. Phys. Lett., 46, pp. L301-L309, 1985. 